\title{
Perfil epidemiológico de pacientes na fila de transplante penetrante de córnea no estado do Pará, Brasil
}

\author{
Epidemiological profile of patients waiting for \\ penetrating keratoplasty in state of Pará, Brazil
}

Edmundo Frota de Almeida Sobrinho', Bianca Caluf Negrão², Hirlana Gomes Almeida²

\section{Resumo}

Objetivo: Descrever o perfil epidemiológico de pacientes na fila de transplante penetrante de córnea no estado do Pará, Brasil. Métodos: Estudo transversal e retrospectivo, com 1261 pacientes, no período de janeiro de 2001 a dezembro de 2009, cadastrados na fila de espera para transplante de córnea na Central de Notificação, Captação e Distribuição de Órgãos (CNCDO) do Pará. Os dados foram coletados por meio de protocolos baseados nas fichas de cadastro de receptor de córnea na CNCDO, adaptados aos objetivos desta pesquisa. Resultados: Em relação à frequência, obteve-se $51 \%$ do sexo masculino; $33,9 \%$ na faixa etária de 61 a 80 anos; $18,6 \%$ inscritos na CNCDO em 2009; $61 \%$ com olho direito acometido; $56 \%$ permaneceram ativos na fila; 51,7\% que aguardaram 1 a 3 anos o transplante e; 85 transplantes realizados em 2009. As principais indicações para transplante de córnea foram ceratopatia bolhosa $(28,2 \%)$, leucoma $(22 \%)$ e úlcera corneana $(14,8 \%)$. Conclusão: Verificou-se que não houve diferença entre a frequência dos sexos sendo que a maioria encontrava-se na faixa etária de 61 a 80 anos; inscrita na CNCDO no ano de 2009 (ano em que foi realizado o maior número de transplantes); com o olho direito acometido, permaneceram ativos na fila de espera. Entre os pacientes transplantados, atestou-se que o tempo em fila de espera para transplante de córnea foi longo e inadequado. Ademais, as principais indicações para transplante de córnea no estado do Pará foram ceratopatia bolhosa, leucoma e úlcera corneana.

Descritores: Transplante de córnea; Ceratoplastia penetrante; Doenças da córnea/diagnóstico; Brasil

\footnotetext{
1 Professor adjunto da Disciplina Oftalmologia da Universidade do Estado do Pará - (UEPA) - Belém (PA), Brasil;

2 Acadêmicas do Curso de Medicina da Universidade do Estado do Pará - (UEPA) - Belém (PA), Brasil.

Trabalho realizado na Central de Notificação, Captação e Distribuição de Órgãos do Pará - Belém (PA) - Brasil

Trabalho recebe auxílio financeiro do Programa Institucional De Bolsas De Iniciação Científica - PIBIC/ CNPq - UEPA

Os autores declaram inexistir conflitos de interesse.
}

Recebido para publicação em: 2/12/2010 - Aceito para publicação em 1/6/2011

Rev Bras Oftalmol. 2011; 70 (6): 384-90 


\begin{abstract}
Purpose: To describe the epidemiological profile of patients waiting for penetrating keratoplasty in state of Pará, Brazil. Methods: Transversal and retrospective study, with 1261 patients, in the period of january 2001 until to december 2009, were registered on the waiting list for corneal transplantation in the Central Notification, Captation and Distribution of Organs (CNCDO) of Pará. Registration forms of cornea receiving were used, adapted to the purposes of this research. Results: In relation to frequency, it was obtained $51 \%$ male; $33.9 \%$ aged 61 to 80 years; $18.6 \%$ enrolled in CNPDO in 2009; $61 \%$ with the right eye affected; $56 \%$ remained active in the list; $51.7 \%$ that waited 1 to 3 years the transplant and; 85 transplants performed in 2009. The main indications for corneal transplantation were bullous keratopathy (28.2\%), scarring (22\%) and corneal ulcer (14.8\%). Conclusion: There was no difference between the frequency of the sexes, the majority were aged 61 to 80 years, entered into the CNPDO in the year 2009 (the year it was held the largest number of transplants), with the right eye was affected, and who remained active in the waiting list. Among the transplanted patients, it was certified that the time in the waiting list for corneal transplantation was long and inappropriate. Furthermore, the main indications for corneal transplantation in state of Pará were bullous keratopathy, scarring and corneal ulcer.
\end{abstract}

Keywords: Corneal transplantation; Keratoplasty penetrating; Corneal diseases/diagnosis; Brazil

\section{INTRODUÇÃO}

As doenças da córnea são causas importantes de cegueira reversível no mundo, atingindo uma população jovem e ativa, o que leva a uma importante perda econômica e social ${ }^{(1)}$.

A falta de tecidos e de Bancos de Olhos capacitados para fornecer córneas em número e qualidade adequados para transplantes é uma realidade, apesar dos esforços realizados ${ }^{(1)}$.

Sabe-se que a Espanha é reconhecida como a maior referência mundial no que diz respeito à captação de órgãos, devido à Organização Nacional de Transplante Espanhola que, prevê a existência em todos os hospitais de uma Comissão Intrahospitalar de Transplantes ${ }^{(2)}$.

O Brasil possui hoje um dos maiores programas públicos de transplantes de órgãos e tecidos do mundo. Com 548 estabelecimentos de saúde e 1.376 equipes médicas autorizadas a realizar transplantes, o Sistema Nacional de Transplantes está presente em 25 estados do país, por meio das Centrais Estaduais de Transplantes ${ }^{(3)}$.

Dados estatísticos mostram que no estado do Pará, até o primeiro semestre de 2009, existiam mais de 900 pacientes aguardando o transplante de córnea, sendo que destes, somente 62 foram realizados ${ }^{(4)}$.

As indicações da ceratoplastia penetrante no Brasil variam em diferentes regiões, sendo o ceratocone a principal indicação na maioria das cidades brasileiras ${ }^{(5-9)}$.

Portanto, considerando que não foram encontrados na literatura científica valores estatísticos que apresentem as principais indicações das ceratoplastias realizadas no estado do Pará, e a grande relevância de se obter dados estatísticos da região Norte, em específico deste estado, o presente estudo objetiva descrever o perfil epidemiológico de pacientes na fila de transplante penetrante de córnea no estado do Pará, Brasil.

\section{MÉTODos}

Todos os prontuários dos pacientes da presente pesquisa foram analisados segundo os preceitos da Declaração de Helsinque e do Código de Nuremberg, respeitando as normas de pesquisa envolvendo seres humanos do Conselho Nacional de Saúde, após aprovação do Comitê de Ética em Pesquisa do Hospital de Clínicas Gaspar Viana e autorizado pelo Coordenador da Central de Notificação, Captação e Distribuição de Órgãos (CNCDO) do Pará.

O estudo realizado foi transversal e retrospectivo. Foram incluídos na pesquisa os pacientes cadastrados na fila de espera para transplante de córnea na CNCDO do Pará, no período de janeiro de 2001 a dezembro de 2009.

Foram excluídos da pesquisa os pacientes que apresentaram dados incompletos na ficha de cadastramento, assim como aqueles que possuíam diagnósticos não condizentes com as reais indicações para transplante, segundo a Portaria $\mathrm{n}^{\circ} 2.600^{(10)}$.

Os dados foram coletados por meio de protocolos baseados nas fichas de cadastro de receptor de córnea na $\mathrm{CNCDO}$, no formato virtual da mesma, adaptados aos objetivos desta pesquisa.

A coleta dos dados foi realizada no período de janeiro a maio de 2010, com o objetivo de identificar as indicações para transplante de córnea no estado do Pará.

Participaram da pesquisa 1271 pacientes enqua- 
drados nos critérios referidos anteriormente, sendo 10 excluídos, totalizando uma casuística de 1261 olhos. Dentre as variáveis, estavam: sexo, data de nascimento, diagnóstico ocular, data de inclusão na CNCDO, situação na lista de espera e data do transplante.

De acordo com a Portaria n ${ }^{\circ} 2.600^{(10)}$, a situação específica de potencial receptor de córnea quanto à manutenção no Cadastro Técnico Único pode ser: ativo, semiativo e removido. Entretanto, neste estudo, foram consideradas as seguintes variáveis quanto à situação na fila de espera: ativo, removido, desistente e transplantado, visto que é fundamental conhecer o número de transplantes realizados, bem como aqueles que optaram por não realizá-lo.

Os dados obtidos foram tratados por métodos da estatística descritiva e comparativa. Para a construção de gráficos e tabelas foram usados os softwares Microsoft Excel $^{\circledR}$ 2007, adotando-se como índice de rejeição da hipótese de nulidade $\mathrm{p}<0,05$, sendo aplicados os Testes Qui-quadrado e Kruskal-Wallis, conforme a indicação, por meio do programa BioEstat $5.0^{\circledR}$.

\section{Resultados}

Foram pesquisados 1261 pacientes cadastrados na CNCDO do estado do Pará no período de janeiro de 2001 a dezembro de 2009 , destes $51 \%$ eram do sexo feminino; $33,9 \%$ situavam-se na faixa etária de 61 a 80 anos; $18,6 \%$ foram inscritos na CNCDO em 2009; 61\% apresentavam o olho direito acometido, e 85 transplantes foram realizados em 2009.

Quanto aos diagnósticos dos pacientes, houve prevalência de ceratopatia bolhosa $(28,2 \%)$, leucoma (22\%) e úlcera (14,8\%) (Tabela 1). Ademais, a análise da situação na fila de espera destes pacientes revelou que $56,2 \%$ permaneceram ativos; $22,9 \%$ foram removidos, $19,6 \%$ transplantados e $1,3 \%$ desistiram de realizar o transplante nesse período (Tabela 2).

Com relação ao tempo de espera destes pacientes, $495(51,7 \%)$ aguardavam de 1 a 3 anos na fila pelo enxerto (Tabela 3 ). Contudo, se cruzada tal variável com a faixa etária, percebe-se que nas faixas etárias mais jovens ( 1 a 20 e 21 a 40 anos) 49,1\% permanecem na fila por até 29 dias (Tabela 4).

Quando comparados o diagnóstico com a faixa etária, a ceratopatia bolhosa se evidenciou em 50,3\% dos pacientes na faixa etária de 61 a 80 e 80 anos ou mais (Tabela 5).

Por sua vez, a relação do diagnóstico versus situação na fila de espera evidenciou que $221(31,2 \%)$ porta-
Tabela 1

Diagnóstico dos pacientes

\begin{tabular}{lcc}
\hline Diagnóstico & Quantidade & \% \\
\hline Ceratopatia bolhosa & 356 & 28,2 \\
Leucoma & 277 & 22 \\
Úlcera corneana & 186 & 14,8 \\
Rejeição ao transplante/ & & \\
falência de enxerto & 169 & 13,4 \\
Opacidade corneana & 95 & 7,5 \\
Ceratocone & 84 & 6,7 \\
Distrofia de fuchs & 43 & 3,4 \\
Descementocele & 30 & 2,4 \\
Outros* & 21 & 1,6 \\
\hline Total & $\mathbf{1 2 6 1}$ & $\mathbf{1 0 0}$ \\
\hline
\end{tabular}

* Ceratite, queimadura química, córnea gutatta, dermóide epibulbar, degeneração corneana macular e estafiloma; (p) Quiquadrado $<0,0001$

Tabela 2

Situação na fila de espera

\begin{tabular}{lcr}
\hline $\begin{array}{l}\text { Situação na fila } \\
\text { De espera }\end{array}$ & Quantidade & \% \\
\hline Ativo & 709 & 56,2 \\
Removido & 289 & 22,9 \\
Transplantado & 247 & 19,6 \\
Desistente & 16 & 1,3 \\
\hline Total & $\mathbf{1 2 6 1}$ & $\mathbf{1 0 0}$
\end{tabular}

(p) Qui-quadrado $<0,0001$

Tabela 3

Tempo de espera na fila

\begin{tabular}{lcc}
\hline Tempo de espera & Quantidade & \% \\
\hline Até 29 dias & 104 & 10,9 \\
1 a 6 meses & 137 & 14,3 \\
7 a 11 meses & 92 & 9,6 \\
1 a 3 anos & 495 & 51,7 \\
4 a 6 anos & 111 & 11,6 \\
7 a 9 anos & 18 & 1,9 \\
\hline Total & $\mathbf{9 5 7}$ & $\mathbf{1 0 0}$
\end{tabular}

(p) Qui-quadrado $<0,0001$ 
Tabela 4

Faixa etária versus Tempo de espera na fila

\begin{tabular}{|c|c|c|c|c|c|c|c|c|c|c|c|c|}
\hline \multirow[t]{3}{*}{ Faixa etária } & \multicolumn{12}{|c|}{ Tempo de espera } \\
\hline & \multicolumn{2}{|c|}{ Até 29 dias } & \multicolumn{2}{|c|}{1 a 6 meses } & \multicolumn{2}{|c|}{7 a 11 meses } & \multicolumn{2}{|c|}{1 a 3 anos } & \multicolumn{2}{|c|}{4 a 6 anos } & \multicolumn{2}{|c|}{7 a 9 anos } \\
\hline & $\mathbf{N}$ & $\%$ & $\mathbf{N}$ & $\%$ & $\mathbf{N}$ & $\%$ & $\mathbf{N}$ & $\%$ & $\mathbf{N}$ & $\%$ & $\mathbf{N}$ & $\%$ \\
\hline 1 a 20 anos & 19 & 18,3 & 12 & 8,8 & 12 & 13,1 & 37 & 7,5 & 6 & 5,4 & 4 & 22,2 \\
\hline 21 a 40 anos & 32 & 30,8 & 30 & 21,9 & 21 & 22,8 & 103 & 20,7 & 29 & 26,1 & 5 & 27,8 \\
\hline 41 a 60 anos & 21 & 20,2 & 27 & 19,7 & 14 & 15,2 & 94 & 19 & 29 & 26,1 & 2 & 11,1 \\
\hline 61 a 80 anos & 28 & 26,9 & 55 & 40,1 & 36 & 39,1 & 183 & 37 & 35 & 31,6 & 5 & 27,8 \\
\hline 80 ou mais & 4 & 3,8 & 13 & 9,5 & 9 & 9,8 & 78 & 15,8 & 12 & 10,8 & 2 & 11,1 \\
\hline Total & 104 & 100 & 137 & 100 & 92 & 100 & 495 & 100 & 111 & 100 & 18 & 100 \\
\hline
\end{tabular}

(p) Kruskal-Wallis $=0.0016$

Tabela 5

Diagnóstico versus Faixa etária dos pacientes

\begin{tabular}{|c|c|c|c|c|c|c|c|c|c|c|}
\hline \multirow[t]{3}{*}{ Diagnóstico } & \multicolumn{10}{|c|}{ Faixa etária (anos) } \\
\hline & \multicolumn{2}{|c|}{1 a 20} & \multicolumn{2}{|c|}{21 a 40} & \multicolumn{2}{|c|}{41 a 60} & \multicolumn{2}{|c|}{61 a 80} & \multicolumn{2}{|c|}{80 ou mais } \\
\hline & $\mathbf{N}$ & $\%$ & $\mathbf{N}$ & $\%$ & $\mathbf{N}$ & $\%$ & $\mathbf{N}$ & $\%$ & $\mathbf{N}$ & $\%$ \\
\hline Ceratopatia bolhosa & 9 & 7,4 & 9 & 3,2 & 33 & 13,3 & 209 & 48,8 & 96 & 53,9 \\
\hline Leucoma & 40 & 32,8 & 79 & 27,7 & 81 & 32,7 & 55 & 12,9 & 22 & 12,4 \\
\hline Úlcera corneana & 27 & 22,1 & 53 & 18,6 & 46 & 18,5 & 49 & 11,4 & 11 & 6,2 \\
\hline Rejeição ao transplante/ falência de enxerto & 8 & 6,6 & 45 & 15,8 & 33 & 13,3 & 51 & 11,9 & 32 & 18,0 \\
\hline Opacidade corneana & 16 & 13,1 & 25 & 8,8 & 23 & 9,3 & 26 & 6,1 & 5 & 2,8 \\
\hline Ceratocone & 15 & 12,3 & 58 & 20,4 & 9 & 3,6 & 1 & 0,2 & 1 & 0,6 \\
\hline Distrofia de fuchs & 1 & 0,8 & 3 & 1,1 & 12 & 4,8 & 20 & 4,7 & 7 & 3,9 \\
\hline Descementocele & 4 & 3,3 & 7 & 2,5 & 7 & 2,8 & 10 & 2,3 & 2 & 1,1 \\
\hline Outros & 2 & 1,6 & 6 & 2,1 & 4 & 1,7 & 7 & 1,7 & 2 & 1,1 \\
\hline Total & 122 & 100 & 285 & 100 & 248 & 100 & 428 & 100 & 178 & 100 \\
\hline
\end{tabular}

(p) Kruskal-Wallis $=0.2807$

Tabela 6

Diagnóstico versus Situação na fila de espera

\begin{tabular}{lcccccccc}
\hline Diagnóstico & \multicolumn{7}{c}{ Situação na fila de espera } \\
\cline { 2 - 9 } & Ativo & \% & Desistência & \% & Removido & \% & Transplantado & \% \\
\hline Ceratopatia bolhosa & 221 & 31,2 & 6 & 37,6 & 87 & 30,1 & 42 & 17 \\
Leucoma & 159 & 22,4 & 4 & 25 & 94 & 32,5 & 20 & 8,1 \\
Úlcera corneana & 66 & 9,3 & 3 & 18,8 & 34 & 11,8 & 83 & 33,6 \\
Rejeição ao transplante/ falência de enxerto & 75 & 10,6 & 2 & 12,4 & 36 & 12,5 & 56 & 22,7 \\
Opacidade corneana & 71 & 10 & 0 & 0 & 13 & 4,5 & 11 & 4,5 \\
Ceratocone & 57 & 8 & 1 & 6,2 & 15 & 5,2 & 11 & 4,5 \\
Distrofia de fuchs & 33 & 4,7 & 0 & 0 & 3 & 1 & 7 & 2,8 \\
Descemetocele & 12 & 1,7 & 0 & 0 & 4 & 1,4 & 14 & 5,6 \\
Outros & 15 & 2,1 & 0 & 0 & 3 & 1 & 3 & 1,2 \\
\hline Total & $\mathbf{7 0 9}$ & $\mathbf{1 0 0}$ & $\mathbf{1 6}$ & $\mathbf{1 0 0}$ & $\mathbf{2 8 9}$ & $\mathbf{1 0 0}$ & $\mathbf{2 4 7}$ & $\mathbf{1 0 0}$ \\
\hline
\end{tabular}

(p) Kruskal-Wallis $=0.002$ 
dores de ceratopatia bolhosa encontravam-se ativos na fila, enquanto que dentre os transplantados $33,6 \%$ apresentavam úlcera corneana (Tabela 6).

\section{Discussão}

O transplante de córnea é o procedimento de maior sucesso entre os transplantes teciduais em humanos e, tem sido o mais realizado na atualidade, tendo como objetivo a restauração da transparência corneana $^{(11)}$.

$\mathrm{Na}$ atual pesquisa, evidenciou-se que a principal indicação de transplante de córnea no estado do Pará foi a ceratopatia bolhosa $(28,2 \%, 326$ olhos), seguida pelo leucoma (22\%, 277 olhos); situando-se e em terceiro lugar a úlcera corneana $(14,8 \%, 186$ olhos) e a falência primária do enxerto ou rejeição ao transplante $(13,4 \%$, 169 olhos) (Tabela 1).

A ceratopatia bolhosa, caracterizada pelo edema corneano estromal, acompanhado de bolhas epiteliais e subepiteliais devido à perda de células e/ou disfunção da função endotelial ${ }^{(12)}$, foi a mais frequente patologia observada como indicação para o transplante, corroborando os estudos realizados em Tokyo por Inoue et al. ${ }^{(13)}$ e em Sergipe por Araújo et al.(14).

Acredita-se que tal prevalência deva-se ao início recente da técnica de facoemulsificação no estado do Pará, ocasionando uma curva de aprendizado que gera um maior número de complicações no pós-operatório, dentre estas a ceratopatia bolhosa.

Este achado diverge de inúmeros estudos realizados no restante do Brasil, na América Latina e na Europa, nos quais o ceratocone desponta como primeira indicação de transplante de córnea ${ }^{(5-9)}$.

Contudo,nesta pesquisa, o ceratocone ocupou o sexto lugar (Tabela 1), devido provavelmente ao não diagnóstico correto desta patologia e acompanhamento necessário, bem como a utilização de anéis intraestromais que retardam a necessidade de transplante ${ }^{(12)}$.

Segundo Araújo et al..$^{(14)}$ e Calix Netto et al. ${ }^{(9)}$, muitos pacientes encaminhados para transplante não apresentam indicação para o mesmo, fato também verificado nesta pesquisa, ao constatar que 1,3\% (considerando os excluídos do estudo) não apresentavam diagnósticos condizentes. Logo, é necessária maior atenção dos profissionais durante a indicação do transplante e preenchimento dos prontuários.

De acordo com a Portaria $\mathrm{n}^{\circ} 2600^{(10)}$, a situação específica de potencial receptor de córnea quanto à manutenção no Cadastro Técnico Único pode ser: ativo (apto e disponível para transplantar), semiativo (com exames pré-transplante incompletos, suspenso pela equipe ou sem condições clínicas) e removido (abandono de tratamento, recusa do transplante, recusas sistemáticas, semiatividade prolongada, removido pela equipe, removido por ausência de condições clínicas, transferido para outro estado, transplantado fora do estado e transplantado com córnea proveniente do exterior).

Entende-se removido por semiatividade prolongada: potenciais receptores inscritos para transplante de córnea, que permaneceram 120 dias cumulativos no status semiativo. Ademais, o removido por recusas sistemáticas é o potencial receptor inscrito que, acumulou cinco recusas por parte da equipe à oferta de tecidos pela $\mathrm{CNCDO}$, de doadores diferentes e em datas distintas ${ }^{(10)}$.

Nesta pesquisa, houve uma prevalência importante de pacientes ativos (Tabela 2), o que demonstra a disparidade entre o número de doadores de córnea e a quantidade de potenciais receptores na lista de espera.

Um tempo de espera prolongado em uma fila para transplante exerce impacto negativo sobre o bem-estar biopsicossocial, as probabilidades de cura, a natureza e extensão das sequelas nos pacientes, nos familiares e na sociedade $^{(15)}$.

Assim, evidenciou-se neste estudo que $51,7 \%$ dos pacientes aguarda de 1 a 3 anos para realizar o transplante de córnea, enquanto que apenas $25,2 \%$ tem o problema resolvido em menos de um semestre (Tabela 3).

A partir desses dados, pode-se observar que no Estado do Pará, os pacientes permanecem por um longo tempo aguardando o transplante. Essa realidade diverge de outros estudos, que evidenciaram uma tendência a homogeneização do tempo de espera em torno de 134 $\operatorname{dias}^{(14,16)}$.

Ao analisar o tempo de espera na fila versus a faixa etária, os pacientes que menos esperam situam-se na faixa etária de 21 a 40 anos e, os que mais esperam são aqueles entre 61 a 80 anos, juntamente com os de 21 a 40 anos (Tabela 4). Tal dado foi estatisticamente significante.

Tal resultado é explicado pela situação de urgência que as patologias que acometem jovens (úlcera, descemetocele e o retransplante) exigem, conferindo prioridade em relação aos demais. Em contrapartida, doenças que acometem idosos são de curso mais arrastado (ceratopatia bolhosa e opacidade corneana), resultando em um maior tempo na fila de espera por um enxerto $^{(12)}$.

O crescimento do número de transplantes de córnea realizado no Pará apresenta inúmeras limitações, o que consequentemente, prolonga o tempo de espera, 
sendo o sistema doação-captação, provavelmente, um dos fatores mais relevantes ${ }^{(16)}$.

Neste estudo, 50,3\% dos pacientes com mais de 60 anos apresentavam ceratopatia bolhosa, e dentre os que se enquadravam na faixa etária mais jovem, a prevalência foi de leucoma $(29,2 \%)$, úlcera corneana $(19,6 \%)$ e ceratocone $(17,9 \%)$ (Tabela 5), concordando com Araújo et al. ${ }^{(14)}$.

Historicamente, sabe-se que a catarata é mais frequente em pacientes com idade superior a 60 anos, sendo realizada para sua correção uma cirurgia que apresenta como principal complicação a ceratopatia bolhosa. Tal fato explica a prevalência deste diagnóstico na faixa etária de 61 a 80 anos e mais de $80 \operatorname{anos}^{(12,14)}$.

Quanto aqueles que apresentavam como indicação o ceratocone, os resultados obtidos concordam com os encontrados por Araujo et al. ${ }^{(17)}$, refletindo o fato desta ser uma patologia que acomete classicamente jovens, com média de idade de 27,4 anos.

No Brasil ${ }^{(18)}$, a fila de transplantes no SUS para cada órgão ou tecido é única, e o atendimento é por ordem de chegada, considerando critérios técnicos, de urgência e geográficos. Segundo Marinho ${ }^{(15)}$, as filas são um resultado do descompasso entre a demanda e a oferta, sendo que a demora no atendimento causa empecilhos na terapêutica.

De acordo com o Department of Health and Human Services do Health Resources and Services Administration (instituição oficial para administração dos transplantes nos Estados Unidos), 70 pessoas são submetidas a transplantes de órgãos por dia nos Estados Unidos. Em contrapartida, 16 pacientes morrem diariamente na fila de espera, evidenciando que uma parcela significativa dos pacientes candidatos a transplantes morre antes do procedimento ser realizado ${ }^{(15)}$.

Já em relação ao Brasil, é importante salientar que ainda não existem dados oficiais disponíveis sobre prazos de espera e mortalidade em filas de transplante ${ }^{(15)}$.

Assim, os períodos de espera são longos e bastante sensíveis às variações na demanda e na oferta de órgãos. Entretanto, existem possibilidades significativas de redução dos prazos nas filas, desde que sejam desenvolvidas políticas internas e externas capazes de incentivar a doação $\mathbf{O}^{(19)}$.

A análise do diagnóstico versus a situação na fila de espera revelou significativamente que a maioria dos pacientes ainda ativos, era portadora de ceratopatia bolhosa, enquanto que a maior parte dos que foram transplantados apresentou úlcera corneana e falência do enxerto ou retransplante (Tabela 6).

Tal resultado é explicado pela situação de urgên- cia que a presença de uma úlcera e de um retransplante exige. Portanto, pacientes portadores destas patologias têm prioridade em relação aos demais. Em contrapartida, a ceratopatia bolhosa, por ser uma doença de curso arrastado, resulta em um maior número de ativos na fila de espera por um enxerto ${ }^{(12)}$.

Em função da grande demanda observada na fila de espera da CNCDO do estado do Pará, sugere-se a capacitação dos profissionais da área da saúde e a realização de campanhas educativas, chamando a atenção para a doação de órgãos.

\section{ConClusão}

Verificou-se que entre os receptores de córnea cadastrados na CNCDO do Pará, não houve diferença entre a frequência dos sexos e, que a maioria encontrava-se na faixa etária de 61 a 80 anos; foi inscrita na CNCDO no ano de 2009 (ano em que foi realizado o maior número de transplantes); com o olho direito acometido pela patologia ocular e, que permaneceram ativos na fila de espera.

Entre os pacientes transplantados, atestou-se que o tempo em fila de espera para transplante de córnea foi longo e inadequado.

Ademais, as principais indicações para transplante de córnea no estado do Pará foram ceratopatia bolhosa, leucoma e úlcera corneana.

\section{Agradecimentos}

As autoras agradecem ao prof. dr.Edmundo Frota de Almeida Sobrinho, professor adjunto da disciplina de Oftalmologia da Universidade do Estado do Pará, por ter disponibilizado seu tempo e conhecimento, sem poupar esforços para a realização deste trabalho e aos funcionários da CNCDO do Pará, que contribuíram com a coleta de dados da pesquisa.

\section{REFERÊNCIAS}

1. Adán CBD, Diniz AR, Sato EH. Dez anos de doação de córneas no Banco de Olhos do Hospital São Paulo: perfil dos doadores de 1996 a 2005. Arq Bras Oftalmol. 2008;71(2):176-81.

2. Matesanz R, Miranda B. A decade of continuous improvement in cadaveric organ donation: the Spanish model. J Nephrol, 2002;15(1):22-8.

3. Brasil. Ministério da Saúde. Legislação sobre o Sistema Nacional de Transplantes. 2009. Brasil. [Internet]. [citado 2009 Set 16]. Disponível em: http://portal.saude.gov.br/portal/ saude/area.cfm?id_area $=1004$.

4. Associação Brasileira de Transplantes de Órgãos. Registro Brasileiro de Transplantes. 2009. [Internet].[citado 2009 Set 16]. Disponível em: http://www.abto.org.br 
5. Endriss D, Cunha F, Ribeiro MP, Toscano J. Ceratoplastias penetrantes realizadas na Fundação Altino Ventura: revisão dos resultados e complicações. Arq Bras Oftalmol. 2003;66(3):273-7.

6. Moreno GL, Souza LB, Freitas D, Sato EH, Vieira LA Transplante de córnea e o conhecimento do procedimento pelos pacientes. Arq Bras Oftalmol, 2003;66(6):797-801.

7. Cattani S, Kwitko S, Kroeff MAH, Marinho D, Rymer S, Bocaccio FL. Indicações de transplante de córnea no Hospital de Clínicas de Porto Alegre. Arq Bras Oftalmol, 2002;65(1):95-8.

8. Flores VGC, Dias HLR, Castro RS. Indicações para ceratoplastia penetrante no Hospital das Clínicas-UNICAMP Arq Bras Oftalmol. 2007;70(3):505-8.

9. Calix Netto MJ, Giustina ED, Ramos GZ, Peccini RFC, Sobrinho M, Souza LB. Principais indicações de transplante penetrante de córnea em um serviço de referência no interior de São Paulo (Sorocaba - SP, Brasil). Arq Bras Oftalmol, São Paulo, 2006;69(5):661-4.

10. Brasil. Ministério da Saúde. Portaria No 2.600 , de 21 de outubro de 2009. Aprova o Regulamento Técnico do Sistema Nacional de Transplantes. Brasil, 21 de outubro de 2009.

11. Chalita MRC, Diazgranados EBM, Sato EH, Branco BC, Freitas D. Rejeição corneana pós transplante de córnea: análise de dados do Banco de Olhos do Hospital São Paulo Escola Paulista de Medicina. Arq Bras Oftalmol. 2000;63(1):55-8.

12. Gonçalves ED, Campos M, Paris F, Gomes JAP, Farias CC. Ceratopatia bolhosa: etiopatogênese e tratamento. Arq Bras Oftalmol; 2008;71(6 Supl 0):61-4.

13. Inoue K, Amano S, Oshika T, Sawa M, Tsuru T. A 10-year review of penetrating keratoplasty. Jpn J Ophthalmol. 2000;44(2):139-45.
14. Araújo AA, Melo GB, Silva RL, Araújo Neta VM. Perfil epidemiológico dos pacientes na lista de espera para transplante de córnea no Estado de Sergipe. Arq Bras Oftalmol. São Paulo, 2004; 67(4):613-6.

15. Marinho A. Um estudo sobre as filas para transplantes no Sistema Único de Saúde brasileiro. Cad Saúde Pública. Rio de Janeiro, out-2006; 22(10):2229-39.

16. Oliveira LA, Corrêa BS, Almeida Junior GC, Ferrari MT, Kashiwabuchi LK. Influência da lista única de uma regional de transplantes de córnea em um Banco de Olhos vinculado a um hospital escola. Arq Bras Oftalmol; 2003;66(5):631-5.

17. Moreira H, Oliveira CS, Godoy G, Wahab SA. Anel intracorneano de Ferrara em ceratocone. Arq Bras Oftalmol, 2002; 65(1):59-63.

18. Brasil. Ministério da Saúde. Portaria ${ }^{\circ} 91 / \mathrm{GM}$ de 23 de janeiro de 2001. [Internet].[citado 2009 Set 16]. Disponível em: http:/ /dtr2001.saude.gov.br/sas/PORTARIAS/Port2001/gM/gm091.htm.

19. Farias RJM, Sousa LB. Impacto do marketing dos processos de divulgação nas doações de córneas a um banco de tecidos oculares humanos e avaliação do perfil socioeconômico de seus doadores. Arq Bras Oftalmol. 2008; 71(1):28-33.

\author{
Endereço para correspondência: \\ Edmundo Frota de Almeida Sobrinho \\ Av. Conselheiro Furtado, $\mathbf{n}^{\circ} 2865$ Ed. Síntese 21, \\ sala 08 e 10 \\ CEP 66063-060 - Belém (PA), Brasil \\ Tel: (91) 3229-3920 \\ E-mail: ealmeida@amazon.com
}

